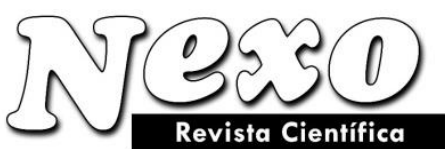

Vol. 33, No. 01, pp. 69-76/Junio 2020
ISSN-E 1995-9516

Universidad Nacional de Ingeniería COPYRIGHT @ (UNI). TODOS LOS DERECHOS RESERVADOS http://revistas.uni.edu.ni/index.php/Nexo https://doi.org/10.5377/nexo.v33i01.10046

\title{
EFECTO DEL EUGENOL EN LA VIDA UTIL DE UNA SALSA DE CHILE JALAPEÑO (Capsicum annum) Y PAPAYA (Carica papaya)
}

\section{EFFECT OF EUGENOL ON THE USEFUL LIFE OF SAUCE FROM CHILE JALAPEÑO (Capsicum annum) AND PAPAYA (Carica papaya)}

\author{
J.A. Marcía Fuentes ${ }^{1, *}$, M.J. Torres Loza ${ }^{1}$ I.M. Varela Murillo ${ }^{1}$, \\ L.A. Chavarría Carrión ${ }^{2}$, H.L. Sanabria Ortega ${ }^{3}$, H.A. Díaz Antúnez ${ }^{3}$ \\ ${ }^{1}$ Universidad Nacional de Agricultura. Facultad de Ciencias Tecnológicas. Catacamas, Honduras. \\ ${ }^{2}$ Universidad Nacional de Ingeniería. Facultad de Ingeniería Química. Managua, Nicaragua. \\ ${ }^{3}$ Universidad Nacional de Agricultura. Laboratorio de Biotecnología. Catacamas, Honduras. \\ juniorabrahamm@yahoo.com
}

(recibido/received: 07-noviembre-2019; aceptado/accepted: 02-abril-2020)

\section{RESUMEN}

Este trabajo tuvo como objetivo medir el efecto del eugenol sobre la vida útil de una salsa picante a partir de chile jalapeño (Capsicum annuum) y papaya (Carica papaya). Se utilizó clavo de olor como material de prueba, empleando diferentes formas de extracción del eugenol como aceite esencial. Se evaluó su uso como preservante en una salsa, mediante métodos acelerados durante 30 días a temperatura de $28^{0} \mathrm{C} \pm 2^{0} \mathrm{C}$ y se comparó con el benzoato de sodio (testigo) según normativa del Codex Alimentarius $(250 \mathrm{mg} / \mathrm{kg})$. Los resultados determinaron que el eugenol presentó efecto inhibitorio del deterioro de la salsa picante prolongado su vida útil, lo que indica su potencial aplicación en la industria alimentaria, convirtiéndolo en una alternativa de conservación natural, cuyo uso ayudaría en la disminución de riesgos toxicológicos aportados por conservantes sintéticos.

Palabras claves: Eugenol; Salsa; Vida útil.

\begin{abstract}
This work aimed to measure the effect of eugenol on the shelf life of a hot sauce from jalapeño pepper (Capsicum annuum) and papaya (Carica papaya). Cloves were tested as test material, using different forms of eugenol extraction as essential oil. Its use as a preservative in a sauce was evaluated by means of accelerated methods for 30 days at a temperature of $280 \mathrm{C} \pm 20 \mathrm{C}$ and was compared with sodium benzoate (control) according to the Codex Alimentarius regulations $(250 \mathrm{mg} / \mathrm{kg}$ ). The results determined that eugenol had an inhibitory effect on the change in hot sauce, prolonged its shelf life, indicating its potential application in the food industry, making it a natural preservation alternative, the use of which will help to decrease the toxicological risks provided by synthetic preservatives.
\end{abstract}

Keywords: Eugenol; Sauce; Useful life.

Nexo Revista Científica / Vol. 33, No. 01, pp. 69-76 / Junio 2020 


\section{J.A. Marcía-Fuentes et al.}

\section{INTRODUCCIÓN}

En general los alimentos presentan una vida útil finita, por lo que necesitan ciertas condiciones de proceso y conservación. Su causa de deterioro es multifactorial, evidenciando pérdida de calidad por microrganismos, por cambios en su composición físico-química y por almacenamiento inadecuado (Díaz, 2009). En la actualidad prolongar la durabilidad de los alimentos garantiza la disminución de pérdidas y propicia mantener la calidad desde un enfoque químico-nutricional y sensorial. Sin embargo, el uso de aditivos químicos como preservantes alimentarios genera una disyuntiva dentro de la comunidad científica, por tanto, algunas sustancias de origen natural empleadas como conservantes han cobrado fuerza e importancia en los últimos años (Burt y Reinder 2003). Además de ser consideradas fuentes de nutracéuticos por su contenido en compuestos bíoactivos y potencial medicinal sobre la salud humana (Marcía et al., 2020).

Según el Reglamento Técnico Centroamericano (2012); una salsa picante se clasifica como un potenciador de sabor por su pungencia, también es considerado un alimento acidificado por su bajo pH. Su deterioro es evidente, principalmente debido a levaduras y Bacterias Ácido Lácticas (BAL), además, puede considerarse el uso de chile jalapeño y otros ingredientes como papaya en su elaboración (Codex, 2017).

Algunos estudios han reportado la acción conservante de los aceites esenciales (AEs) en alimentos, como potencial competencia de agentes preservantes sintéticos que no certifican su inocuidad (Goñi et al., 2009).

Los AEs son una mezcla de lípidos o grasas de bajo peso molecular muy hidrofóbicas, generalmente menos densos que el agua, son aromáticos y volátiles, producto del metabolismo secundario de las plantas, formados por terpenos, en particular monoterpenos y sesquiterpenos, así como sus derivados oxigenados, alcoholes, aldehídos, ácidos y ésteres terpénicos que se denominan respectivamente, monoterpenoides y sesquiterpenoides (Pereira et al., 2008: Rivas et al., 2015). Tienen la ventaja de ser obtenidos de materiales vegetales y se ha demostrado su inocuidad en humanos, por ejemplo, los AEs de hierbas, especias y clavo de olor (Goñi et al., 2009). Los AEs de clavo de olor y canela poseen actividades biológicas antibacterianas, antifúngicas, insecticidas y antioxidantes, atribuidas al eugenol y al aldehído cinámico, se utilizan tradicionalmente como saborizantes y como antimicrobianos en los alimentos (Guan et al., 2007; Affonso et al., 2012; Fuentes et al., 2020).

El Eugenol es un derivado fenólico conocido comúnmente como esencia de clavo, que también puede extraerse de pimienta, hojas de laurel, canela, alcanfor y otros aceites. Es de consistencia líquida y aceitosa, de color amarillo claro, con aroma característico, poco soluble en agua y soluble en alcohol (Gonzales, 2002). Castallo (2012); evaluó la actividad inhibitoria de los aceites esenciales de clavo de olor (Syzygium aromaticum) y canela (Cinnamomum verum), en un estudio in vitro e in vivo sobre la levadura Rhodotorula mucilaginosa, en leche chocolatada. Además, se estudió el efecto del eugenol sobre la vida útil en productos de panadería, obteniendo una durabilidad de 57 días (Pilco et al., 2009).

Teniendo en cuenta los elementos anteriormente expuestos, se plantea el presente trabajo con el objetivo medir el efecto del eugenol sobre la vida útil de una salsa picante a partir de chile jalapeño (Capsicum annuum) y papaya (Carica papaya)

\section{MATERIALES Y MÉTODOS}

Para la extracción del aceite esencial, se empleó como materia prima $1 \mathrm{~kg}$ de clavo de olor de tipo comercial, se procedió a maceración manual, segmentándose en unidades experimentales de $100 \mathrm{~g}$ dentro de matraz aforado de $200 \mathrm{ml}$ tipo Pyrex para cada unidad. Se utilizó como disolvente agua y

Nexo Revista Científica / Vol. 33, No. 01, pp. 69-76 / Junio 2020 


\section{J.A. Marcía-Fuentes et al.}

soluciones hidro-alcohólicas (etanol-agua) al $60 \%, 70 \%$ y $80 \%$, con relación de 1 a 5 respectivamente (100 g de clavo de olor en $500 \mathrm{ml}$ de disolvente), además se empleó el método de destilación por arrastre de vapor utilizando etanol al $97 \%$. Para la elaboración de la salsa picante, se empleó como materia prima; $1 \mathrm{~kg}$ de chile jalapeño variedad Papantla, $2 \mathrm{~kg}$ de fruta bomba o papaya variedad Puna, $100 \mathrm{~g}$ de sal común (cloruro de sodio), 5 litros de agua potabilizada y 3 litros de vinagre comercial (ácido acético).

Mediante un diseño de mezcla optimizado con el programa Desing Expert versión 11.0 se obtuvieron las diferentes formulaciones, empleando para su proceso una licuadora tipo semi-industrial marca Oster motor de $26000 \mathrm{rpm}$ con capacidad de 3 litros, una estufa eléctrica marca Whirlpool de 4 quemadores, dos termómetros marcan Upate international a doble escala y utensilios de cocina de acero inoxidable. Las formulaciones para la prueba se obtuvieron a partir de la mezcla de chile jalapeño y concentrado de papaya con mínimos y máximos del $30 \%$ al $60 \%$, sal común (cloruro de sodio) del $0.5 \%$ al $1 \%$, vinagre comercial (ácido acético) entre $5 \%$ y $20 \%$ y eugenol entre $0 \%$ y $10 \%$ como conservante de prueba. Se utilizó un modelo de vértices, con puntos centrales y tres réplicas de control, para un total de 22 unidades experimentales que fueron segmentadas en muestras de $100 \mathrm{~g}$. Estas unidades se evaluaron sensorialmente con pruebas hedónicas de 7 puntos a escala de laboratorio, en 50 jueces de tipo afectivo con edades comprendidas entre 20 años y 50 años de edad, siguiendo la metodología ISO 6658:2008 propuesta por Espinoza (2014) y Marcía et al., (2019). La fórmula con mayor aceptación se empleó para el estudio de la durabilidad.

Se determinó su vida útil en muestras de $100 \mathrm{~g}$ de salsa picante por métodos acelerados, a tiempos de $0,7,14,21,28$ y 30 días desde su elaboración, a temperatura de almacenamiento de $28^{0} \mathrm{C} \pm 2^{0} \mathrm{C}$. Se tomó como variable de respuesta la calidad físico-química que incluyó: determinación de $\mathrm{pH}$, viscosidad y ${ }^{\circ}$ Brix

Para obtener una mejor interpretación de los hallazgos, se utilizó la metodología con ligeras modificaciones de Marcía et al., (2019); a partir del uso de análisis estadístico, en relación a las formulaciones de prueba y el análisis sensorial, utilizando el programa IBM, SPSS, versión 25.0

\section{RESULTADO Y DISCUSIÓN}

La tabla 1 resume que el eugenol presentó un nivel de extracción aceptable en soluciones hidroalcohólicas del 60 al 80\%. Estos datos coinciden con lo expresado por Pinto (2018); que indica que relaciones hidro-alcohólicas arriba del $70 \%$, son más estables para extraer este aceite esencial. Sin embargo, requiere de refrigeración durante 24 h, filtrado en frío, reposo por 72 h y decantación para su clarificación. Se descarta el uso de agua desmineralizada para la obtención del eugenol y la técnica de destilación por arrastre de vapor, a partir de etanol al 97\%, es la mejor técnica para extraerlo empleando temperaturas comprendidas entre $70^{\circ} \mathrm{C}$ y $120^{\circ} \mathrm{C}$, durante $4 \mathrm{~h}$ y $2 \mathrm{~h}$ respectivamente.

Tabla 1. Características de extracción del eugenol (100g)

\begin{tabular}{ccccc}
\hline$\%$ Etanol & $\% \mathrm{H}_{2} \mathrm{O}$ & $\mathrm{pH}$ & ${ }^{\circ}$ Brix & Tipo de extracción \\
\hline 0 & 100 & 6.9 & 0 & Ninguno \\
60 & 40 & 4.6 & 36 & Medio \\
70 & 30 & 4.7 & 39 & Medio \\
80 & 20 & 4.9 & 45 & Medio \\
\hline
\end{tabular}

Nexo Revista Científica / Vol. 33, No. 01, pp. 69-76 / Junio 2020 


\section{J.A. Marcía-Fuentes et al.}

La tabla 2 resume que el eugenol es un fluido que presenta condiciones organolépticas únicas en su tipo, con un olor característico a su fuente, un olor amarillo y un sabor pungente, validando lo expuesto por Castaño (2012); en relación a las características sensoriales del eugenol obtenido a partir de clavo de olor.

Tabla 2. Descripción sensorial del eugenol

\begin{tabular}{cc}
\hline Descriptor & Especificaciones \\
\hline Olor & Muy aromático \\
Color & Amarillo \\
Sabor & Pungente \\
Aspecto general & Fluido \\
\hline
\end{tabular}

A partir del análisis sensorial de preferencia de consumo, se determinó que las formulaciones con eugenol del $0 \%$ al $3 \%$ obtuvieron aceptación, sin embargo, formulaciones entre $4 \%$ y $10 \%$ fueron rechazadas. Estos resultados coinciden con la investigación de Pinto (2018); que indica que concentraciones de eugenol, mayores del $3 \%$ en alimentos manifiestan rechazo.

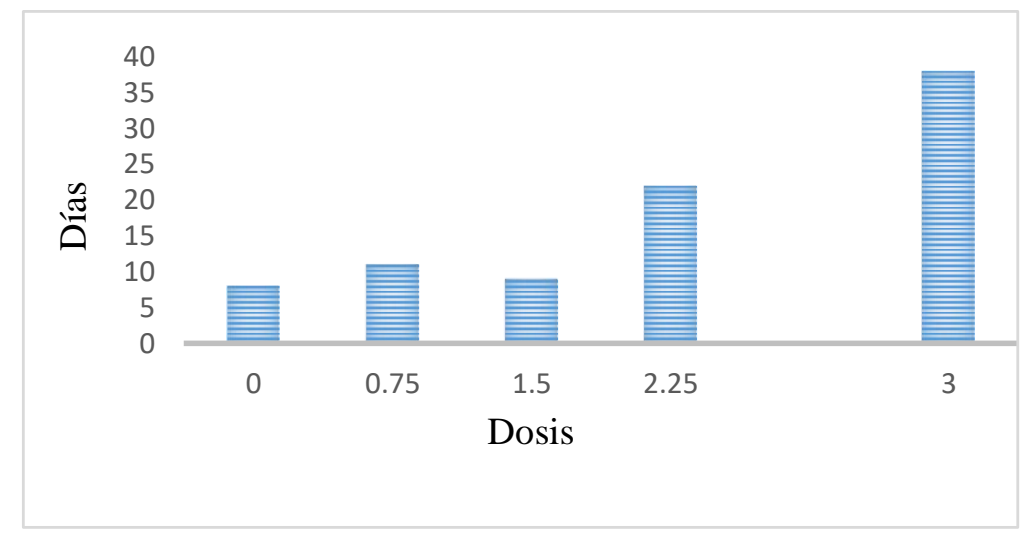

Figura 1. Durabilidad de la salsa picante respecto a dosis de eugenol empleada

La Figura 1 resume que las concentraciones de eugenol del $3 \%$ en adelante, mostraron un efecto inhibitorio sobre el deterioro de la salsa y valores por debajo del $3 \%$ evidenciaron un alto deterioro microbiano (Figura 2). Estos resultados coinciden por lo expuesto por Flores y Aryana (2018) y Fuentes et al., (2020); que manifestó inhibición de BAL en concentraciones de eugenol mayores al $3 \%$.

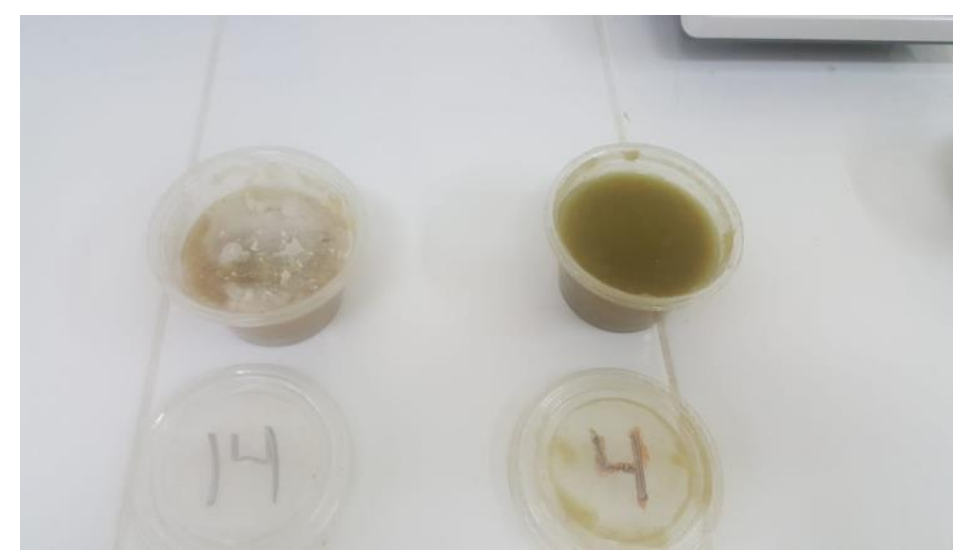

Figura 2. Presencia de hongos en formulaciones con eugenol menor al 3\%

Nexo Revista Científica / Vol. 33, No. 01, pp. 69-76 / Junio 2020 


\section{J.A. Marcía-Fuentes et al.}

El uso de eugenol al $3 \%$ en el diseño de mezcla, se consideró la formula optimizada por su buena aceptación sensorial y su efecto inhibitorio en el deterioro de la salsa. Siendo esta la concentración que se utilizó para el cálculo de la durabilidad.

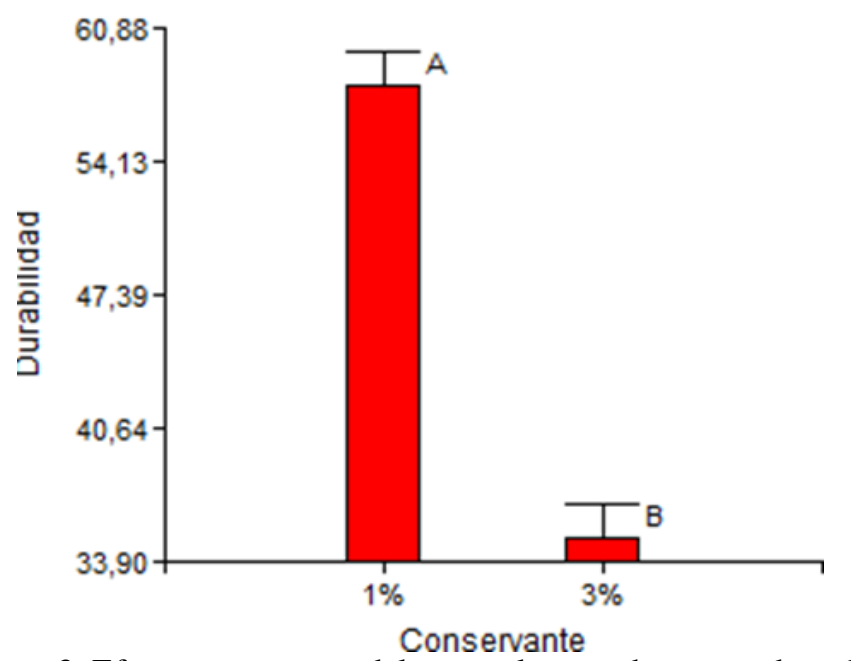

Figura 3. Efecto conservante del eugenol versus benzoato de sodio

La figura 3 muestra los resultados obtenidos del efecto conservante del eugenol (B) respecto al benzoato de sodio (A), sobre la vida útil de la formulación con mayor aceptación sensorial. Partiendo de una reacción de primer orden, con un nivel de tratamiento al $3 \%$ de eugenol y una cinética de deterioro de $0.066357 \mathrm{~d}^{-1}$ a partir de las variables físico-químicas de control de $\mathrm{pH}$, solidos solubles y viscosidad, se determinó que la durabilidad de esta formulación es de 45 días para la pérdida total de sus atributos, sin embargo, el limite critico se evidencia en la perdida de color al día 35. Con respecto al control el benzoato de sodio presentó una durabilidad mucho mayor, alcanzando el limite critico en el color hasta los 58 días, estableciendo una diferencia de 23 días en relación al empleo del eugenol al $3 \%$, además mostro mayor estabilidad en las variables de control (Figura 4).

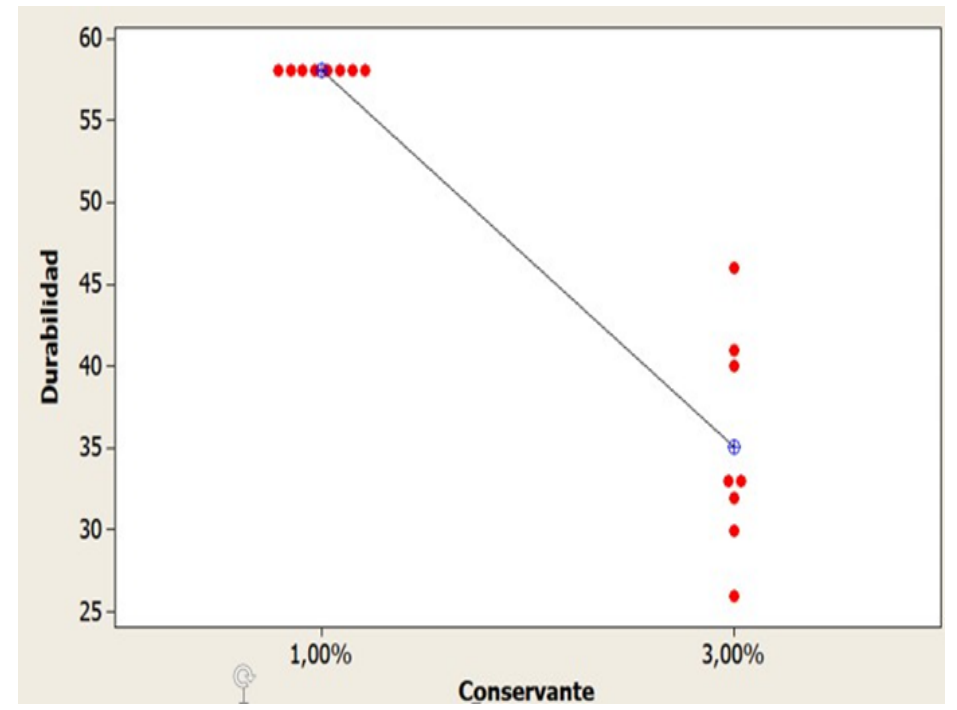

Figura 4. Comparación de la estabilidad de los valores físico-químico entre las pruebas

Nexo Revista Científica / Vol. 33, No. 01, pp. 69-76 / Junio 2020 


\section{J.A. Marcía-Fuentes et al.}

\section{CONCLUSIONES}

Los mejores resultados del eugenol como conservante en una salsa picante, se obtuvo a partir de una fórmula optimizada de chile jalapeño al $31 \%, 60 \%$ papaya, $5 \%$ vinagre (ácido acético), $1 \%$ sal (cloruro de sodio) y $3 \%$ eugenol. Las formulaciones por debajo del $1 \%$ de este aceite esencial, mostraron un deterioro acelerado en la calidad físico-química y sensorial, además de una marcada presencia de hongos al corto tiempo.

El eugenol como agente conservante en una salsa picante, mostro su efecto antimicótico y retardante de la cinética de deterioro, lo que justifica su potencial aplicación en la industria de alimentos y su posible uso como sustituto de preservantes sintéticos, de bajo costo y fácil extracción.

El mayor rendimiento de extracción del eugenol como aceite esencial de clavo de olor, es a partir de la destilación por arrastre de vapor a $120^{\circ} \mathrm{C}$ durante 2 horas.

\section{REFERENCIAS}

Affonso, R.S., Rennó, M.N., Slana, G.B., França, T.C. (2012). Chemical and Biological Aspects of the Essential Oil of Indian cloves. Revista Virtual de Química, 4 (2), 146-161.

Burt, S.A. \& Reinders, R.D. 2003. Antibacterial activity of selected plant essential oils against Escherichia coli O157: H7. Lett. Appl. Microbiol, 36 (1), 162-167.

Castaño, M. (2012). Evaluación de la capacidad conservante de los aceites esenciales de clavo (Syzygiumaromaticum) y canela (Cinnamomum verum), sobre la levadura (Rhodotorula mucilaginosa) en leche chocolatada, (en línea). (Tesis inédita de ingeniería). Universidad Nacional de Colombia, Medellín, Colombia.

Codex Alimentarius. (2017). Norma regional para la salsa de ají (chiles). CXS 306R-2011.

Díaz Torres, R. (2009). Conservación de alimentos. La Habana, Cuba: Editorial Universitaria Felix Varela.

Espinoza Manfugás, M. (2014). Análisis sensorial. La Habana, Cuba: Editorial Universitaria Felix Varela.

Flores Soler, R. \& Aryana, K. (2018). Influence of cloves on viability of Lactobacillus acidophilus. School of Animal and Food Sciencie. Louisiana State University.

Fuentes J.A.M., Fernández I.M., Alemán R.S., Maldonado S.A.S., Flores Soler R., Funez N.H., Chavarría L.A, \& Kayanush A. (2020). Chemical Characterization of the Essential Oil of Syzygium Aromaticum and its Antimicrobial Activity Against A Probiotic Lactobacillus Acidophilus. European Scientific Journal, ESJ, 16 (15), 33.

González Escobar, Raimara. (2002). Eugenol: propiedades farmacológicas y toxicológicas. Ventajas y desventajas de su uso. Revista Cubana de Estomatología, 39 (2), 139-156.

Nexo Revista Científica / Vol. 33, No. 01, pp. 69-76 / Junio 2020 


\section{J.A. Marcía-Fuentes et al.}

Goñi, P., López, P., Sánchez, C., Gómez, R., Becerril, R., Nerín, C. (2009). Antimicrobial activity in the vapor phase of a combination cinnamon and clove essential oils. Food Chemistry, 11 (6), 982-989.

Guan, W., Li, S., Yan, R., Tang, S., Quan, C. (2007). Comparison of clove bud essential oils extracted with supercritical carbon dioxide and three other traditional extraction methods. Food Chemestry, 101 (4), 1558-1564.

Marcía Fuentes, J., Chavarría Carrión, L., \& Zumbado, H. (2019). Análisis del proceso de harina de yuca, sobre las propiedades sensoriales y nutricionales del casabe (Artículo Profesional). Nexo Revista Científica, 32(01), 88-93.

Marcía Fuentes, J., Montero Fernández, I., Zumbado, H., Lozano Sánchez, J., Santos Alemán, R., Navarro Alarcon, M., Borrás Linares, I., Saravia Maldonado, S. (2020). Quantification of Bioactive Molecules, Minerals and Bromatological Analysis in Carao (Cassia grandis). Journal of Agricultural Science, 12 (3), 88-94.

Pereira, A.A.; Cardoso das Graças, M.; de Morais, L.R.; de Guimaraes, A.R.; de Lima, L.G. \& Salgado, A.P.S.P. (2008). Caracterização química e efeito inibitório de óleos essenciais sobre o crescimento de Staphylococcus aureus e Escherichia coli. Ciência e Agrotecnologia, 32 (3), 887-93.

Pilco, S., Quito, M., Quispe, S. (2009). Conservación de pan artesanal Ezequiel y pan super bueno usando aceite esencial de clavo de olor (Eugenia caryophillus). Revista de investigación Universitaria. 1 (1), 2-6.

Pinto, B. (2018). Efecto del eugenol sobre la durabilidad de frijoles procesados. (Tesis inédita de ingeniería). Universidad Nacional de Agricultura, Olancho, Honduras.

Reglamento Técnico Centroamericano, RTCA. (2012). Normativa RTCA 67.04.54:10 para alimentos y bebidas procesadas: aditivos alimentarios.

Rivas, K.; Rivas, C.; Gamboa, L. (2015). Chemical composition and antimicrobial activity of basil essential oil (Ocimum basilicum L.). Rev., Multiciences, 15 (3), 281-289.

\section{SEMBLANZA DE LOS AUTORES}

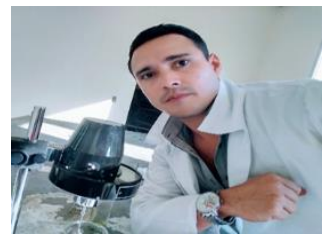

Jhunior Abrahan Marcía Fuentes: Ingeniero Agroindustrial de la Universidad Nacional Autónoma de Honduras. Master en Procesamiento de Alimentos en la Universidad Nacional de Ingeniería de Managua, Nicaragua. Candidato a Doctor en el grado Científico en Alimentos en la Universidad de La Habana, Cuba. Actualmente se desempeña como profesor de la Universidad Nacional de Agricultura de Honduras (UNA), en la Facultad de Ciencias Tecnológicas, en la carrera de Ingeniería en Alimentos. Ganador del Premio Joven Investigador Latinoamericano 2019, del Grupo COIMBRA de Universidades Europeas. Investigador colaborativo de prestigiosas Universidades y Centros de Investigación entre las que destacan Louisiana State University de los Estados Unidos, El Centro de Investigación para el Desarrollo del Alimento Funcional y Nutracéutico, CIDAF y la Universidad de Granada, España. En la actualidad desarrolla investigaciones en plantas nativas del continente americano, en las que destacan plantas del Amazona de Brasil y Honduras, para su potencial uso como nutracéuticos y bíoconservantes alimenticios. 


\section{J.A. Marcía-Fuentes et al.}

Leonardo Chavarría Carrión, Ingeniero Químico (UNI, 2000). Master en Procesamiento de Alimentos (UNI-2003), Master en Gestión Universitaria (UNI, 2009) coautor del artículo, Investigador en las áreas de: Desarrollo de Nuevos Productos y Seguridad Alimentaria y Nutricional, Profesor titular de la Facultad de Ingeniería Química de la Universidad Nacional de Ingeniería (UNI) Nicaragua, Director del Programa de Vinculación e Innovación Tecnológica (PVIT). 